\title{
Face recognition using enhancement discrete wavelet transform based on MATLAB
}

\author{
Asma Abdulelah Abdulrahman, Fouad Shaker Tahir \\ Department of Applied Sciences, University of Technology, Baghdad, Iraq
}

\begin{tabular}{l} 
Article Info \\
\hline Article history: \\
Received Feb 3, 2021 \\
Revised May 17, 2021 \\
Accepted Jun 21, 2021 \\
\hline Keywords: \\
Bit pere pixel \\
Compression ratio \\
Convolution neural network \\
De-noise \\
Discrete chebyshev wavelet \\
transform \\
Face recognition \\
Mean square error
\end{tabular}

\begin{abstract}
In this work, it was proposed to compress the color image after de-noise by proposing a coding for the discrete transport of new wavelets called discrete chebysheve wavelet transduction (DCHWT) and linking it to a neural network that relies on the convolutional neural network to compress the color image. The aim of this work is to find an effective method for face recognition, which is to raise the noise and compress the image in convolutional neural networks to remove the noise that caused the image while it was being transmitted in the communication network. The work results of the algorithm were calculated by calculating the peak signal to noise ratio (PSNR), mean square error (MSE), compression ratio (CR) and bit-per-pixel (BPP) of the compressed image after a color image $(256 \times 256)$ was entered to demonstrate the quality and efficiency of the proposed algorithm in this work. The result obtained by using a convolutional neural network with new wavelets is to provide a better CR with the ratio of PSNR to be a high value that increases the high-quality ratio of the compressed image to be ready for face recognition.
\end{abstract}

This is an open access article under the CC BY-SA license.

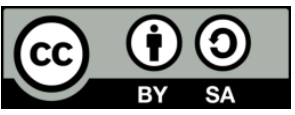

\section{Corresponding Author:}

Asma Abdulelah Abdulrahman

Department of Applied Sciences

University of Technology, Baghdad, Iraq

Email: Asma.A.Abdulrahman@uotechnology.edu.iq

\section{INTRODUCTION}

There are many methods of identifying people and identifying them, such as by fingerprint, eye print, facial recognition, and its parts. The fingerprint technology emerged to identify the person because the fingerprint of a person belongs to the person alone because it is not repeated by another person [1] but this technique may be unclear, here the ability of deep learning appears, especially the convolutional neural network system because the network convolutional neural network (CNN) is multilayered, resulting in organically extruded contrast [2]. An inquiry appears as to which deep learning strategies are ideal Ismael and Irina [3]. The eye print is also one of the methods of detecting a person's identity. As for the face recognition process, the face recognition system is a computer device that has the ability to recognize the person in the form of a digital image from the video, face recognition using Viola-Jones depending on new technology programming [4]. Using face recognition technology using Python technology, the face object, and the rest of the objects in the database, so that the system decides to allow the person to enter or not [5]. Studies reveal the recognition of parts of the face such as the nose, eyes, and mouth. This is an important development in facial recognition. A consistent Viola-Jones algorithm has been used to detect objects and engineer the facial parts of the image from recent studies [6]-[10].

The Wavelets such as Haar in open CV programming technology were used to create a viola. Input is a group of images to format an algorithm in the extensible markup language (XML) file for facial 
recognition and eye detection in a specific image. Haar wavelengths of the image were used using the Adaboost algorithm to collect weak classifiers and produce a strong classifier data set, which led to the speed and accuracy of recognition the face [11]-[15]. A good and fast video-based human face recognition algorithm has been proposed [8]. A MATLAB-based convolutional neural network approach for face recognition system facial recognition was performed using MATLAB, with skin type identification and convolutional neural network (CNN) training [16]. The role of wavelets is highlighted in image processing, the use of matlab, and many important algorithms [17]-[20]. The training of convolutional neural networks has taken its role in many works that have been identified and studied and their role in image processing and face recognition and their use in the fields of medicine, airport security and wishful thinking [21]-[25]. In this work, a new fast wavelet discrete chebyshev wavelet transform (DCHWT) was used to analyze the image and de-noise to be compressed, and then train the convolutional neural network after raising the noise to which the image was exposed during the conversion in order to obtain the best and fastest results. A single sample and optimization with DCHWT will be used in Figure 1.
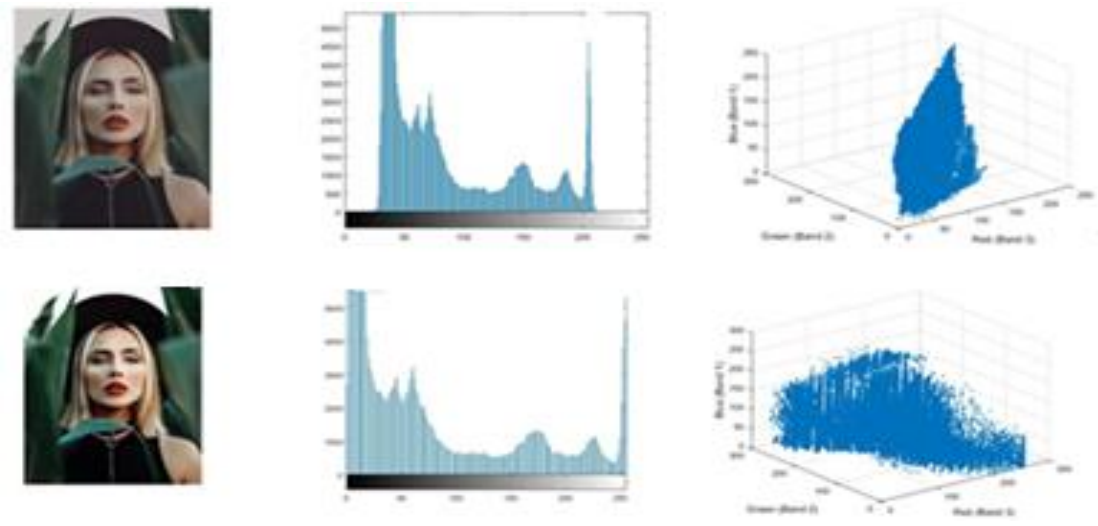

Figure 1. Enhancement color image with DCHWT

\section{RESEARCH METHOD}

The aim of this work was to using effect of new wavelets DCHWT in analyzing the color image, after which the image is subjected to an image optimization process to get rid of unwanted distortion. Color feature extraction is performed on enhanced images. The enhanced images are converted and compressed and the neural network trained for the purpose of detecting the face, but before sending the image to the network the noise that the image may be exposed to during transmission is raised to obtain desirable results. Figure 2 represents the steps involved in this work. Table 1 illustrates the performance of the steps of reach to biter results.

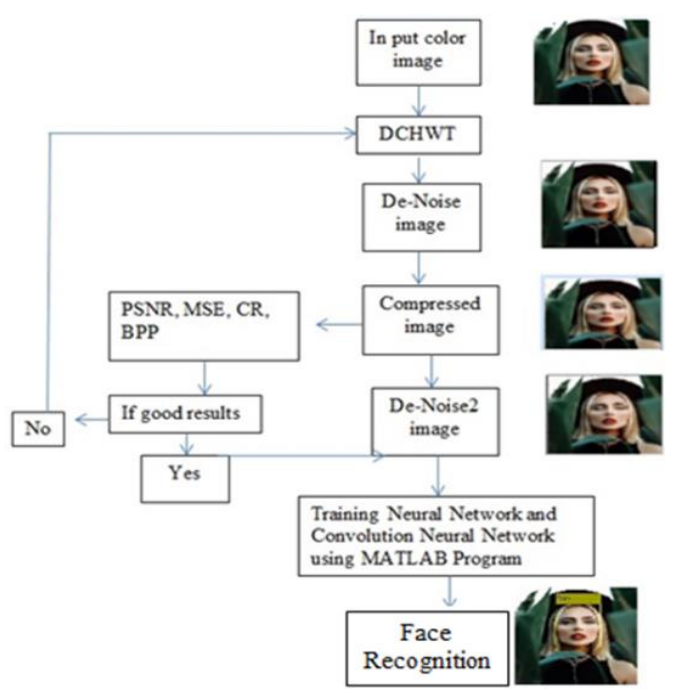

Figure 2. A suggested flowchart for identifying the face recognition problem 
Table 1. The performance of the steps of reach to biter results

\begin{tabular}{|c|c|c|c|c|c|}
\hline loop & Image compression steps & MSE & PSNR & BPP & $\mathrm{CR}$ \\
\hline 1 & & 10000.51 & 6.341 & 0.0070 & $0.03 \%$ \\
\hline 2 & & 10000.12 & 7.639 & 0.0073 & $0.03 \%$ \\
\hline 3 & & 5247 & 10.93 & 0.0083 & $0.03 \%$ \\
\hline 4 & & 4601 & 11.5 & 0.0089 & $0.04 \%$ \\
\hline 5 & & 3450 & 12.75 & 0.0124 & $0.05 \%$ \\
\hline 6 & & 2534 & 14.09 & 0.0228 & $0.10 \%$ \\
\hline 7 & & 1428 & 16.58 & 0.059 & $0.25 \%$ \\
\hline 8 & & 827 & 18.96 & 0.149 & $0.62 \%$ \\
\hline 9 & & 351.7 & 22.67 & 0.384 & $1.60 \%$ \\
\hline 10 & & 143.6 & 26.56 & 0.778 & $3.24 \%$ \\
\hline 11 & & 56.55 & 30.61 & 1.409 & $5.87 \%$ \\
\hline 12 & & 22.37 & 34.63 & 2.328 & $9.70 \%$ \\
\hline 13 & & 8.848 & 38.66 & 3.631 & $15.13 \%$ \\
\hline 14 & & 3.978 & 42.13 & 5.344 & $22.2 \%$ \\
\hline 15 & & 2.587 & 44 & 7.310 & $30.46 \%$ \\
\hline 16 & & 2.251 & 44.61 & 9.593 & $39.97 \%$ \\
\hline
\end{tabular}




\subsection{Discrete chebyshev wavelet transform (DCHWT)}

A lot of research included how to construct the wavelet, which consists of the mother function, where the movement depends on two important coefficients a and b, through which the extension and the first translation is responsible for the extension and the second of the translation where the process is continuing on this case:

$$
\Psi_{a, b}(x)=|a|^{\frac{-1}{2}} \Psi\left[\frac{x-b}{a}\right] a, b \in R, a \neq 0
$$

where $\psi(t)=\left[\psi_{0}(t), \psi_{1}(t), \ldots, \psi_{M-1}\right]^{T}$.

The elements $\psi_{0}(t), \psi_{1}(t), \ldots, \psi_{M-1}(t)$ are the basis functions, orthogonal on the [0,1]. Let dilation by parameter $a=2^{-k}$, translation by parameter $b=(a(2 n-1))$ and transform $x=a\left(2 a^{-1} t\right)$, by substitute parameters a, b and transform $\mathrm{x}$ in (1), then will be get (2). DCHWT $T_{n, m}(t)=T(k, n, m, t)$ include four parameters, $n=1,2, \ldots, 2^{k} \mathrm{k}$ is:

$$
T_{n, m}(t)=\left\{\begin{array}{cl}
\frac{\sigma_{m} 2^{\frac{k}{2}}}{\sqrt{\pi}} T_{m}\left(2^{k+1} t-2 n+1\right) & \frac{n-1}{2^{k}} \leq t<\frac{n}{2^{k}} \\
0 & \text { otherwise }
\end{array}\right.
$$

Where $\sigma_{m}=\left\{\begin{array}{cc}\sqrt{2} & m=0 \\ 2 & m=1,2, \ldots\end{array}\right.$

The new used of proposed wavelets are applicable in analyzing the color image in both the horizontal and vertical directions and by using the wavelet filter through its low and high passes on the rows of the image and then on the columns separately. Figure 3 represents the process of analyzing the image, which shows the division of the image into four parts, LL is the approximate coefficients, HL, LH, and $\mathrm{HH}$ they are represented details coefficients.

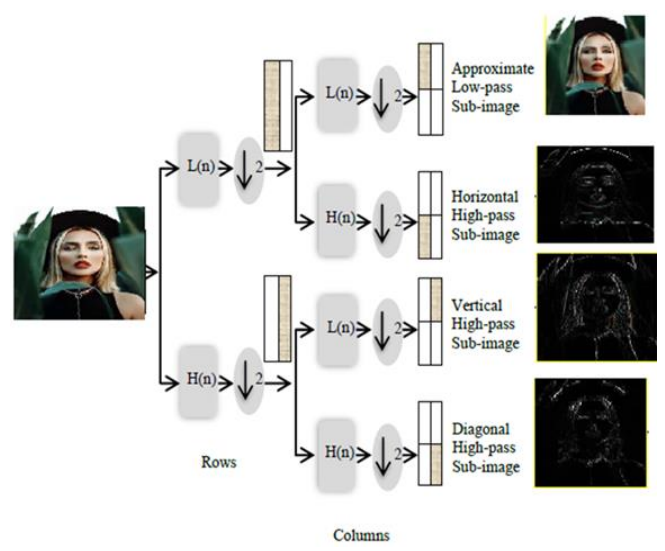

Figure 3. represents the process of analyzing the image

\subsection{De noise image with DCHWT}

Lifting noise from the color image with noise, to preserve image features while removing noise, waves are used, resulting in a sparse representation of the image, which means that the wave transmission focuses on the features of the image by dealing with the large-scale parameters of the wave. For small wave parameters, the parameters will be reduced and defined or eliminated without affecting image quality. After that, the process begins. Reconstruct the image data using the reverse wavelet transformation, Figure 4 shows the image after turning off the noise.

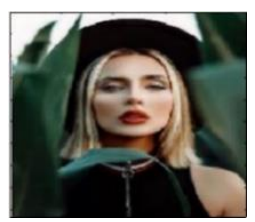

Figure 4. De noise color image with DCHWT 


\subsection{Compassion image with DCHWT}

By discrete chebyshev wavelet transformation (DCHWT), the compression is achieved by image decomposition, because the new wavelets have the characteristic of orthogonality in the first group after that by quantitative technique is applied to the approximate coefficients and binary coding parameters, after which the number of pixels is reduced, which leads to an increase in the rate compression. The following metrics that determine image quality after ideal compression are applied:

- Mean squared error (MSE) is the difference between the original and the reconstructed image pixels defined as [10]:

$$
M S E=\frac{1}{M N} \sum_{i=1}^{M} \sum_{j=1}^{N}\left(x_{i j}-y_{j i}\right)^{2}
$$

- Peak signal to noise ratio (PSNR) measures the perceptual quality of the image compressed on the term of distortion defined by [11], [13]:

$$
P S N S=10 \log _{10}\left(\frac{255^{2}}{M S E}\right)
$$

- Compression ratio (CR) is:

$$
C R=\left(1-\frac{N}{M}\right) \times 100
$$

Where N: number of pixels in compressed image.

$\mathrm{M}$ : number of pixels in original image.

- Bit per pixel (B.P.P) gives the number of bits used to store one pixel of the image. For a grayscale image, the initial BPP is 8 bits [10]-[13].

$$
B P P=\frac{\text { Compressed image size in bits }}{\text { Total number of pixels in the image }}
$$

After the compression process, the image is sent for facial recognition during the transmission process, and some noise may be attached to the image, which calls for a noise-raising operation that has the whole image using the wavelets so that the face is recognized using the theory of convolutional neural networks Figure 5 is compressed color image with DCHWT.

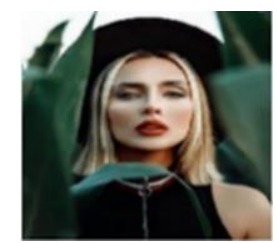

Figure 5. Compressed color image with DCHWT

\subsection{Wavelet convolution neural network (W-CNN)}

The artificial neural network performs a work similar to the work of the human brain, the basic building block that is called a neuron or unit, so that the information is received from the outside, which is called the input. The connection point between every two neurons represents the weight that creates the network's layers. The characteristic that the wavelet possesses is similar to the neural network of the layer $\left(1+\frac{1}{2}\right)$ that has one or more inputs by passing through the hidden layer. Either one or more output layers have activated the wavelet function to form neurons. To overcome the limitations of the mentioned techniques, a convolutional neural network was used to overcome the limitations of the aforementioned techniques that were used. To classify the face, which was in two stages, the training step is to extract the feature of the face and adjust the weight of the neural networks after reducing the dimensions. The second step is to recognize the face that was not previously recognized.

\subsection{Mathematical of training convolutional neural network}

The filter is singled to center each pixel in the filter that must contain the kernel $\mathrm{N}$ with the number of channels identical to the image, so a different filter is applied that passes on each channel, in (3) calculates the dimensions of the filter. 


$$
\text { Dim filter }=(F, F, n c)
$$

The image is mapped with filter the convolutional process mathematically is:

$$
\begin{aligned}
& C(I, K)_{x, y}=\sum_{i=1}^{n_{H}} \sum_{j=1}^{n_{W}} \sum_{K=1}^{n_{C}} K_{i, j-K} I_{x+i-1, y+j-1, K} \\
& \operatorname{dim}(C(I, K))=\left(\left[\frac{n_{H}+2 P-F}{S}+1\right]\right) S>0 ;
\end{aligned}
$$

If $S=1$ in (5) will be

$$
\left(n_{H}+2 P-F, n_{W}+2 P-F\right)
$$

Where [n] is the floor function of $\mathrm{n} P=0$ is the valid CNN output size is equal output size then $P=\frac{F-1}{2}$ is the same $\mathrm{CNN}$, if $\mathrm{F}=1$, this mean $1 \times 1$ Convolution. The merging and reduction process distinguishes the image by specifying its information on each channel, which affects the dimensions $\left(m_{H}, m_{W}\right)$ and maintains the number of channels after passing the filter.

$$
\left.\operatorname{dim}(P(I, K))=\left(\left[\frac{n_{H}+2 P-F}{S}+1\right], \frac{n_{W}+2 P-F}{S}+1\right], n_{C}\right) \quad S>0
$$

If $S=1$ in (5) will be

$$
\left(n_{H}+2 P-F, n_{W}+2 P-F, n_{C}\right.
$$

After the training process, using CNN leads to the reduction of a number of layers Figure 6 shows how a process $\mathrm{F}$ led to the reduction of the three layers.

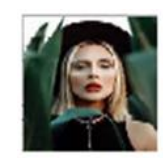

De noise

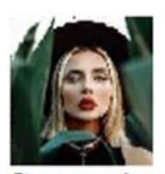

Compassion
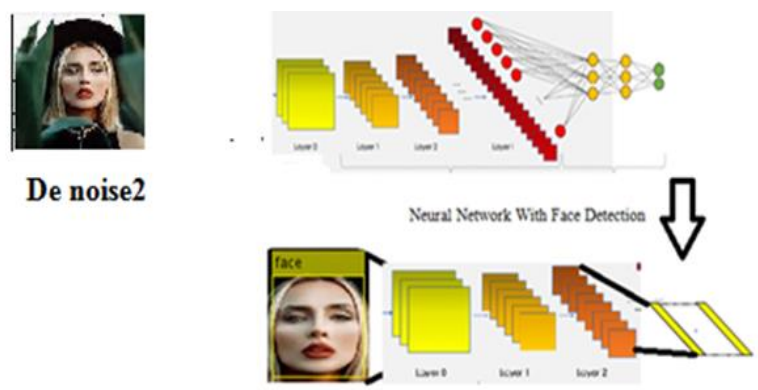

Figure 6. represents the convolutional network training process to detect a face with DCHWT

\subsection{Face recognition}

Facial recognition technology is one of the complex applications and it has great importance at the same time with computer vision and to obtain the best results, the MATLAB program is used for its accuracy and speed, and in this work more than one technique was used to obtain high-precision results. The image was analyzed into the three basic layers and the image was compressed with DCHWT and with convolutional neural network training for face recognition via the CascadeObjectDetector function included in the MATLAB toolbox applications.

\subsection{Face recognition algorithm with DCHWT and CNN}

A fast algorithm for face detection with a new technology, which is the use of wavelets to analyze the image into the three basic layers, red, green, and blue, and work on improving the image. A convolutional neural network was trained after compressing the image using the suggested wavelets. Figure 6 represents the convolutional network training process to detect a face with DCHWT and Figure 7 shows the results of programming the algorithm for facial recognition with DCHWT and CNN. Figure 8 shows how to use the MATLAB program to identify the face. The proposed algorithm, with the help of MATLAB, to identify the face, the following steps show the stages of the face recognition the result of the proposed algorithm. 
Algorithm with DCHWT and CNN for face recognition

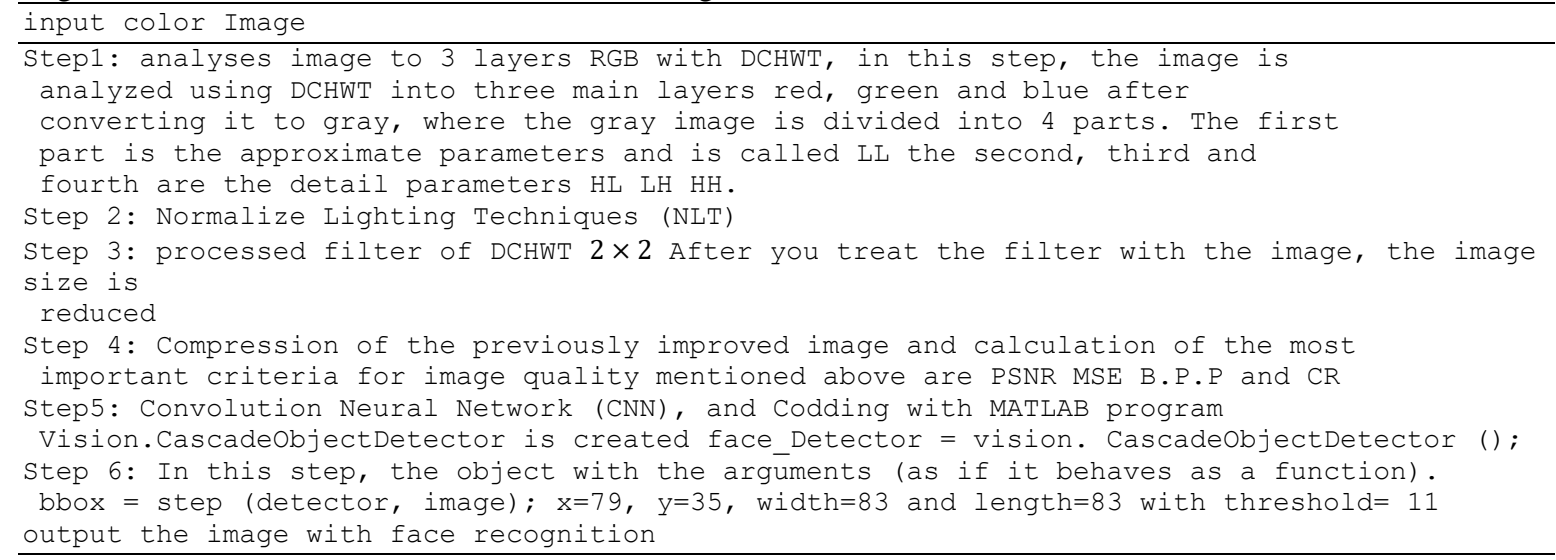

\begin{tabular}{ll}
$\boxplus$ a & $127 \times 169 \times 3$ vint8 \\
$\boxplus$ bbox & {$[62,27,65,65]$} \\
\hline detector & $1 \times 1$ CascadeObjectD... \\
$\boxplus$ videoOut & $127 \times 169 \times 3$ vint8
\end{tabular}
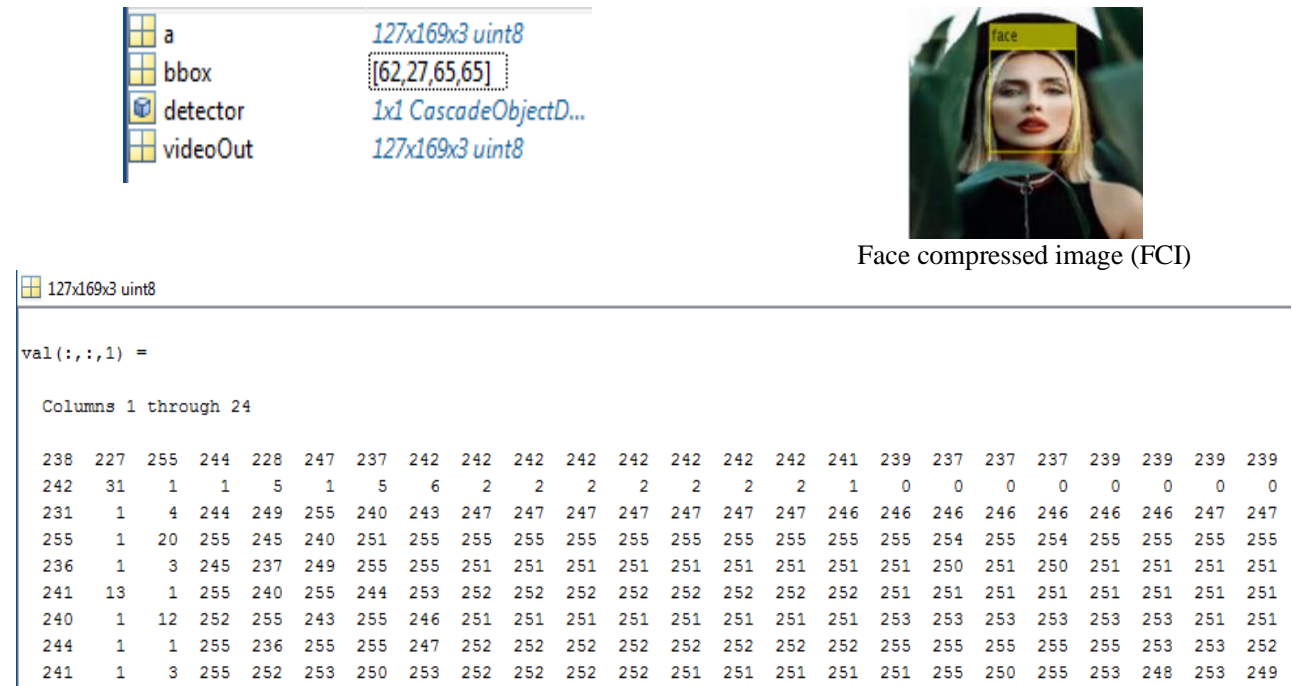

Figure 7. Shows the results of programming the algorithm for facial recognition with DCHWT and CNN

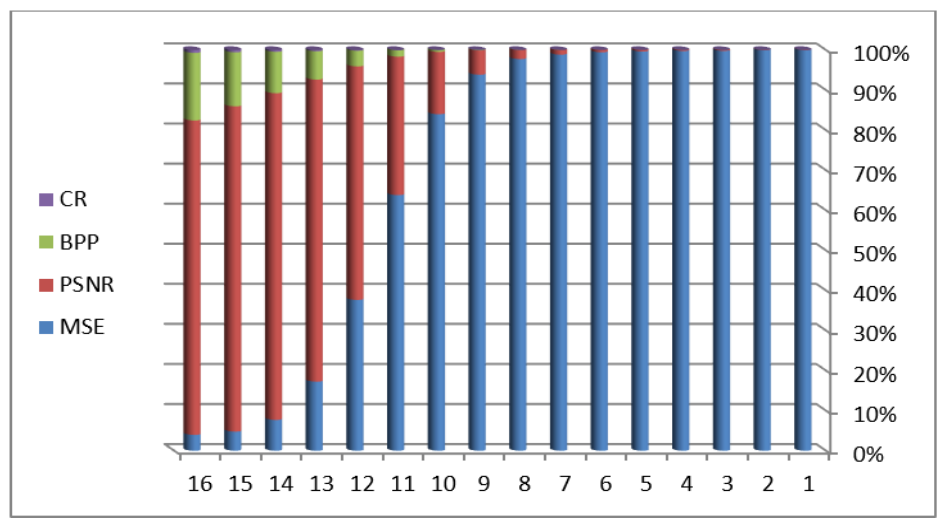

Figure 8. Illustrated chart the values of PSNR, MSE, BPP, and CR

\section{RESULTS AND DISCUSSION}

Using the new wavelets with the convolutional neural network training to provide the $3 \times 3$ filter and raise the noise so that the image is compressed before the face is detected using the MATLAB program and in the Table 1 the case of compression improvement is studied step by step and how the peak signal increases due to reducing the error rate that leads to an increase PSNR With the compression rate from the 
table, it becomes clear in the first step that MSE is very large, reaching 10000.51 corresponding to a small amount in PSNR, and respectively the second and third steps until to reach step 16 is a significant improvement in the results $\mathrm{MSE}=2.251$, $\mathrm{PSNR}=44.61, \mathrm{BPP}=9.593$, and $\mathrm{CR}=39.97 \%$ which leads to the quality of the compressed image free from noise to face recognition with inside a box with the title to reveal the features of the face $[62,27,65,65]$ mean $x=62 y=27$ length and width are equal 65 with threshold 1 to be inside the box that is shown in the Figure 7 and Figure 8 illustrated values of PSNR, MSE, BPP and CR.

\section{CONCLUSION}

For face recognition using the new wavelets with the training of the convolutional neural network, in this work, many errors, and ambiguities were reduced in which face recognition was done using a new technology of wavelets to analyze the image, raise the noise and then compress it, and the best results were obtained to reduce the error and raise the quality of the resulting image. Training a convolutional neural network for facial recognition using MATLAB and computer intelligence to make use of this technology in disease recognition and medical image processing.

\section{REFERENCES}

[1] H. Mohamed and K. Wang, "Parallel score fusion of ECG and fingerprint for human authentication based on convolution neural network," Computers \& Security, vol. 81, pp. 107-122, March 2019, doi: 10.1016/j.cose.2018.11.003.

[2] W. J. Wong and Shang-Hong Lai, "Multi-task CNN for restoring corrupted fingerprint images," Pattern Recognition, vol. 101, P. 107203, May 2020, doi: 10.1016/j.patcog.2020.107203.

[3] K. D. Ismael and S. Irina, "Face recognition using Viola-Jones depending on Python," Indonesian Journal of Electrical Engineering and Computer Science (IJEECS), vol. 20, no. 3, pp. 1513-1521, December 2020, doi: 10.11591/ijeecs.v20.i3.pp1513-1521.

[4] T. S. Arulananth, M. Baskar, and R. Sateesh. "Human face detection and recognition using contour generation and matching algorithm," Indonesian Journal of Electrical Engineering and Computer Science (IJEECS), vol. 16, no. 2, pp. 709-714, November 2019, doi: 10.11591/ijeecs.v16.i2.pp709-714.

[5] A. M. A. Hossen, R. A. A. Ogla, and M. M. Ali. "Face Detection by Using OpenCV's Viola-Jones Algorithm based on coding eyes," Iraqi Journal of Science, vol. 58, no. 2A, pp. 735-745, 2017.

[6] R. Singh and M. Kaur, "Face Recognition and Detection using Viola-Jones and Cross Correlation Method." International Journal of Science and Research (IJSR), vol. 4, no. 1, pp. 2498- 2501, Jan. 2015.

[7] R. I. Bendjillali, M. Beladgham, K. Merit, and A. Taleb-Ahmed, "Illumination-robust face recognition based on deep convolutional neural networks architecture," Indonesian Journal of Electrical Engineering and Computer Science (IJEECS), vol. 18, no. 2, pp. 1015-1027, May 2020, doi: 10.11591/ijeecs.v18.i2.pp1015-1027.

[8] Syazana-Itqan K, Syafeeza A.R* and Saad N.M, "A MATLAB-Based Convolutional Neural Network Approach for Face Recognition System," Journal of Bioinformatics and Proteomics Review, vol. 2, no. 1, pp. 1-5, 2016, doi: 10.15436/2381-0793.16.009.

[9] M. Rasheed, A. Abdulelah, and S. Shihab, "The Effect of Set Partitioning in Hierarchical Trees with Wavelet Decomposition Levels Algorithm for Image Compression," Electronics Science Technology and Application, vol. 7 no. 3, pp. 40-46, 2020.

[10] G. Al-Khafaji and H. Al-Mahmood, "Lossless Compression of Medical Images using Multiresolution Polynomial Approximation Model," International Journal of Computer Applications, vol. 76, no. 3, pp. 38-42, 2013, doi: 10.5120/13230-0659.

[11] S. Aruna Deepthi, E. Sreenivasa Rao, and M. N. Giri Prasad, "RTL implementation of image compression techniques in WSN," International Journal of Electrical and Computer Engineering (IJECE), vol. 9, no. 3, pp. 1750-1756, 2019, doi: 10.11591/ijece.v9i3.pp1750-1756.

[12] A. M. Hadia and A. A. Abdulrahman, "Image compression based with multi discrete Laguerre wavelets transform," Journal of Southwest Jiaotong University, vol. 55, no. 2, pp. 1-17, 2020, doi: 10.35741/issn.0258-2724.55.2.45.

[13] F. S. T. Al-Azawi and A. A. Abdulrahman, "Face Detection By some Methods based on MATLAB," J. of AlQadisiyah for Comp. Sci. and Math., vol. 12, no. 4, pp. 12-17, 2020, doi: 10.29304/jqcm.2020.12.4.712.

[14] E. Setyaningsih and A. Harjoko "Survey of Hybrid Image compression Techniques," International Journal of Electrical and Computer Engineering (IJECE), vol. 7, no. 4, pp. 2206-2214, 2017, doi: 10.11591/ijece.v7i4.pp22062214.

[15] A. A. Abdulrahman, M. S. Rasheed, and S. N. Shihab, "The Analytic of Image Processing Smoothing Spaces Using Wavelet," Journal of Physics: Conference Series, vol. 1879, no. 022118, pp:1-15, 2021.

[16] S. laith, A. A. Abdulrahman, and F. S. T. Al-Azawi, "Face Detection for Color Image Based on MATLAB," Published in Journal of Physics Conference Series, vol 1879, no. 022129, pp:1-10, 2021.

[17] S. L. Galib, F. S. Tahir, and A. A. Abdulrahman, "Detection Face parts in image using Neural Network Based on MATLAB," Engineering and Technology Journal, vol. 39, no. 1, pp. 159-164, 2021, doi: 10.30684/etj.v39i1B.1944. 
[18] K. Panimozhi and G. Mahadevan, "QoS Frame work for a multi stack based heterogenous Wireless sensor network," International journal of Electrical and Computer Engineering (IJECE), vol. 7, no. 5, pp. 2713-2720, 2017, doi: 10.11591/ijece.v7i5.pp2713-2720.

[19] B. Mostafa, C. Saad, and H. Abderrahmane, "Firefly algorithm to improve threshold distributed energy efficient clustering algorithm for heterogenous Wireless sensor Networks," IAES International Journal of Artificial Intelligence, vol. 6, no. 3, pp. 91-99, 2017, doi: 10.11591/ijai.v6.i3.pp91-99.

[20] T Karras, S Laine and T Aila, "A Style Based Generator Architecture for Generative Adversarial Networks," Proceedings of the IEEE/CVF Conference on Computer Vision and Pattern Recognition (CVPR), Long Beach CA USA, 2019, pp 4396-4405, doi: 10.1109/CVPR.2019.00453.

[21] K. Syazana-Itqan, A. R. Syafeeza, and N. M. Saad, "A MATLAB-Based Convolutional Neural Network Approach for Face Recognition System," Journal of Bioinformatics, Proteomics and Image Analysis, Vol. 2, no. 1, pp. 71-75, 2016, doi: 10.15436/2381-0793.16.009.

[22] K. Chen, C. Liu, and Y. Xu, "Face Detection and Tracking Based on Adaboost CamShift and Kalman Filter Algorithm," Computational Intelligence, Networked Systems and Their Applications. Springer, Berlin, Heidelberg, 2014. 149-158, doi: 10.1007/978-3-662-45261-5_16.

[23] A. S. Abdallah, A. L. Abbott, and M. A. El-Nasr, "A New Face Detection Technique using 2D DCT and Self Organizing Feature Map," Proceedings of World Academy of Science, Engineering and Technology, vol. 21, 2007, pp: $15-19$.

[24] C. Zhang, Z. Zhang, S. Dickinson, and G. Medioni, "Boosting-based face detection and adaptation Synthesis Lectures," in Face Detection and Adaptation, California, USA: Morgan and Claypool Publishers, 2010, pp: 1-140.

[25] G Ravindra, "Dabhade Optimal Neural Network Based Face Recognition System for Various Pose and Occluded Images," International Journal of Applied Engineering Research, vol. 12, no. 22, pp: 12625-12636, 2017.

\section{BIOGRAPHIES OF AUTHORS}
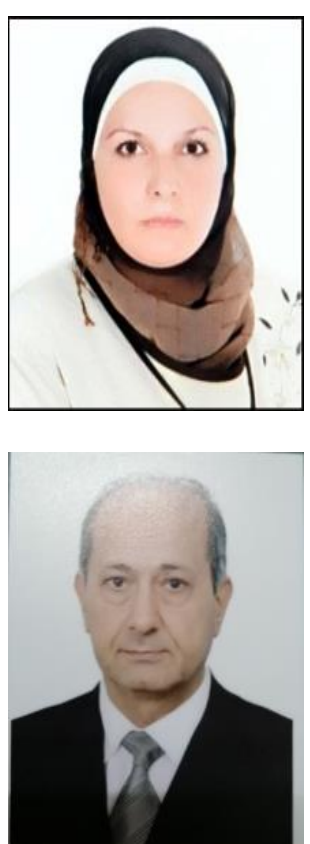

Asma Abdulelah Abdulrahman she was born in Iraq-Baghda. She works at Applied Sciences, University of Technology/BaghdadApplied Siences, University of Technology/Baghdad. Languages spoken Arabic-English, Ph.D. Image processing/Applied Mathema Acharya Nagarjuna University 2019 MSc. Optimal control Method, Applied Mathematics, University of Technology 2012. BS Department of Mathematics University of Technology 2007. More than ten research papers have been published in the field of applied mathematics. Fifteen papers have been published in the field of image processing and neural networks and Artificial intelligence.

Fouad Shaker Tahir he was born in Iraq-Baghda. He works at Applied Sciences, University of Technology/Baghdad Applied Siences, University of Technology/Baghdad. Languages spoken Arabic-English, Ph.D. Artificial intelligence Algorithms and digital systems. University of Technology 2004, MSc. Applied Mathematics, University of Technology 1998. BS Electrical Engineering, University of Technology 1984. More than ten research papers have been published in the field of applied mathematics. Fifteen papers have been published in the field of image processing and neural networks and Artificial intelligence. 